\title{
Blood Pressure and Cognitive Decline in Prevalent Hemodialysis Patients
}

\author{
David A. Drew ${ }^{a}$ Hocine Tighiouart $^{b}$ e Sarah Duncan $^{a} \quad$ Jasmine Rollins $^{a}$ \\ Aditi Gupta ${ }^{c}$ Tammy Scott ${ }^{d}$ Daniel E. Weiner ${ }^{a}$ Mark J. Sarnak ${ }^{a}$ \\ ${ }^{a}$ Division of Nephrology, Department of Medicine, Tufts Medical Center, Boston, MA, USA; ${ }^{b}$ The Institute for

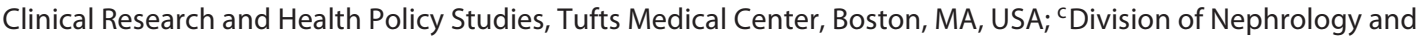 \\ Hypertension, Department of Internal Medicine, University of Kansas Medical Center, Kansas City, KS, USA; ${ }^{d}$ Jean \\ Mayer USDA Human Nutrition Research Center on Aging at Tufts University, Boston, MA, USA; 'Tufts Clinical and \\ Translational Science Institute, Tufts University, Boston, MA, USA
}

\section{Keywords}

Hemodialysis · Blood pressure · Systolic · Diastolic ·

Cognitive impairment

\begin{abstract}
Background: Hypertension is associated with cognitive decline in the general population. It is unclear what impact blood pressure (BP) has on cognitive decline in patients receiving maintenance hemodialysis (HD). Methods: Using a longitudinal cohort of 314 prevalent HD patients without dementia at baseline, we examined the association of predialysis systolic BP (SBP) and diastolic BP (DBP), pulse pressure, and intradialytic SBP change (pre minus post), averaged for a month, with cognitive decline. Cognitive function was determined by a neurocognitive battery, administered yearly. Individual cognitive test results were reduced into 2 domain scores using principal components analysis (by definition mean of 0 and SD of 1), representing memory and executive function. Joint models, allowing for characterization of cog-
\end{abstract}

nitive score slopes and including adjustment for potential confounders, were utilized to account for competing risks from death, dropout, or kidney transplantation. Results: Mean age was 62 years; $54 \%$ were men, $23 \%$ were black, and $90 \%$ had at least a high school education. During median follow-up of 2.1 years (25th-75th: 1.0-4.5), 191 had at least one follow-up test, 148 died, and 43 received kidney transplants. Low predialysis DBP and high pulse pressure were both associated with steeper executive function decline (each $10 \mathrm{~mm} \mathrm{Hg}$ lower DBP $=-0.03 \mathrm{SD}$ [-0.01 to -0.05$]$ per year steeper decline) in executive function (each $10 \mathrm{~mm} \mathrm{Hg}$ higher pulse pressure $=-0.03 \mathrm{SD}[-0.06$ to -0.01$]$ steeper decline) but not for memory function. SBP and intradialytic change were not associated with steeper decline for either memory or executive function. Conclusions: No relationship was seen between SBP or intradialytic change in BP with cognitive decline. In prevalent HD patients, lower predialysis DBP and wider predialysis pulse pressure are associated with steeper cognitive decline in executive function but not memory.

\section{KARGER}

(c) 2019 S. Karger AG, Basel

E-Mail karger@karger.com

www.karger.com/ajn
David A. Drew, MD MS

Division of Nephrology, Department of Medicine

Tufts Medical Center, 800 Washington Street

Box 391, Boston, MA 02111 (USA)

E-Mail ddrew@ tuftsmedicalcenter.org 


\section{Introduction}

Studies of blood pressure (BP) in the general population demonstrate associations between higher systolic BP (SBP) and diastolic BP (DBP) and cognitive impairment [1-4]. Patients with chronic kidney disease (CKD) often have concomitant hypertension, potentially placing them at high risk for cognitive impairment [5]. Those with kidney failure requiring dialysis have an even higher prevalence of cognitive impairment with $20-60 \%$ demonstrating cognitive deficits [6], with the majority of dialysis patients also displaying hypertension.

There is significant controversy over appropriate BP targets in the general population [7]. This uncertainty extends to patients with kidney disease [8], a population that may benefit from intensive BP targets [9], while also being more susceptible to adverse outcomes from aggressive $\mathrm{BP}$ control [10]. Additional ambiguity arises from the aging related fall in DBP and associated loss of vascular elasticity, which raises the question as to the clinical impact of low DBP. Unfortunately, minimal data are available to support BP targets in hemodialysis (HD) populations [11]. Importantly, there are only a few studies of BP and cognitive impairment in patients with $\operatorname{CKD}[5,12]$, and none have evaluated the longitudinal relationship between specific BP measures and changes in cognitive function.

We previously demonstrated an association between low DBP and high pulse pressure with impaired executive function (a cognitive domain closely linked with cerebrovascular disease) in cross-sectional analyses; however, these associations were attenuated after multivariable adjustment [12]. Based on these cross-sectional findings and previously described longitudinal associations between BP and cognitive function in nondialysis populations, we evaluated the association between predialysis systolic and DBP as well as pulse pressure with cognitive decline in a cohort of maintenance HD patients, focusing on memory and executive function cognitive domains [6, 13].

\section{Materials and Methods}

\section{Study Population}

Outpatients 18 years old or older receiving maintenance incenter HD at 5 Dialysis Clinic Inc. (DCI) units and one hospitalbased outpatient unit (St. Elizabeth's Medical Center) in the greater Boston area were screened for the Cognition and Dialysis Study, with study enrollment occurring from January 28, 2004, to May 31, 2012 [6]. Eligibility criteria included English fluency as well as sufficient visual and hearing acuity to complete neurocognitive testing. To minimize floor effects and to maximize the likelihood of completing the full battery of tests, individuals with mini-mental state examination (MMSE) score $\leq 10$ and/or possible diagnosis of dementia were excluded. Determination of whether a participant had possible dementia was based on a detailed review of the dialysis unit and hospital electronic record for any mention of dementia. Additional exclusion criteria included nonaccess related hospitalization within 1 month of screening, receipt of $\mathrm{HD}$ for $<1$ month, and single pool (sp) Kt/V $<1.0$. The Tufts Medical Center/Tufts University Institutional Review Board approved this study, and all participants who agreed to undergo detailed cognitive testing signed informed consent. The clinical and research activities reported are consistent with the Declaration of Helsinki.

\section{Baseline Demographics and Clinical Characteristics}

Demographic, clinical, and laboratory factors were ascertained at the time of cognitive testing. Education $(<12$ th grade, high school graduate to $<2$ years of college, and $\geq 2$ years of college) was obtained via patient questionnaire. History of cardiovascular disease (CVD), defined as a composite of either coronary artery disease and/or peripheral vascular disease, was determined by patient history or documentation in the patient's electronic or paper chart. Patients were queried about personal history of myocardial infarction and coronary revascularization, which were used to define coronary disease, and about intermittent claudication and peripheral vascular disease, which were used to define peripheral vascular disease. Additionally, DCI electronic medical and paper records were reviewed for a history of these conditions, with specific focus on problem lists, hospital discharge summaries, cardiac test results, and procedure results. Additional medical history including primary cause of ESRD, HD vascular access type, and dialysis vintage (time since HD initiation) were obtained from the DCI or St. Elizabeth's electronic record. Serum albumin and spKt/V most proximate to the time of cognitive testing were obtained from participant medical records.

\section{Blood Pressure}

For all BP measures, the monthly average at the time of baseline cognitive testing was utilized. Systolic and DBP readings were obtained in the outpatient dialysis unit as a part of usual clinical care immediately prior to the start as well as immediately after completion of HD sessions and were obtained from the DCI electronic records. Per dialysis unit protocols, nurses and technicians are instructed to measure BP in the nonaccess arm with an appropriately sized cuff, using an automated system, prior to the initiation of the HD session. Pulse pressure was obtained by subtracting DBP from SBP. Intradialytic change in BP was defined as the difference between predialysis SBP and postdialysis SBP.

\section{Neurocognitive Assessment}

At study enrollment, participants were administered a battery of neurocognitive tests by research coordinators after a period of training and direct observation by the study neuropsychologist (Dr. Tammy Scott). The same battery of tests was administered yearly to study participants whenever possible. To maintain quality and inter-rater reliability, testing was observed by the study neuropsychologist at 3-6 months intervals. To limit subject fatigue, all testing was completed during the 1st hour of HD. Using 
the same battery of tests, we have previously demonstrated similar performance regardless of whether testing was performed during the 1st hour of dialysis or before the start of dialysis [14]. When possible, neurocognitive testing was performed in a private room or in as quiet an environment as possible. The neurocognitive battery included well-validated commonly used cognitive tests (online suppl. Table 1; for all online suppl. material, see www.karger. com/doi/10.1159/000500041) that possess high inter- and intrarater reliability. The MMSE was used as a screening test [15]. The neurocognitive battery consisted of the Wechsler Memory ScaleIII Word List Learning Subtest [16], the Wechsler Adult Intelligence Scale-III Block Design [16] and Digit Symbol-Coding Subtests [16], and Trail Making Tests A and B (Trails A and B) [17]. For Trails B, a 300 second time limit was imposed, with those unable to complete the test during this time period considered "noncompleters." In year 3 of the study, the cognitive panel was expanded to include additional verbal tests assessing both memory and executive functions, including Digit Span (forwards and backwards) [16], the Mental Alternation Test [18], and the Controlled Oral Word Association Test [19].

Our prespecified primary outcomes were the association of each baseline BP measure with change in memory and executive function over time. Principal component analysis (PCA) with varimax rotation was used as a data reduction technique with 292 participants to derive composite scores for separate cognitive domains (memory or executive function) in the entire study population [20]. For 18 individuals who were missing baseline results on one cognitive test (or 2 results if derived from the same test), single item imputation was performed using multivariable linear regression models based on performance on other tests in the cognitive battery. Two principal components with eigenvalues $>2$ were obtained (Component 1 eigenvalue $=2.87$, Component 2 eigenvalue $=2.29$ ), and the resulting component scores subsequently were used as coprimary outcomes. Using this method, all component scores have a mean of 0 and SD of 1 . The first component was interpreted to reflect executive functioning, attention, and processing speed (referred to as executive function in the Results section), with the Trails A and B, Block Design, and Digit SymbolCoding tests contributing significantly (online suppl. Table 1). The second component primarily was composed of Word List Learning Recall and Recognition and was interpreted to reflect memory. Component loadings for deriving the principal component score at the baseline examination $(n=292)$ were used to calculate the principal component scores for follow-up testing, which included 8 patients who did not have enough data to calculate PCA scores at baseline, bringing the number of unique participants up to 300 . Digit Span, Mental Alternations and the Controlled Oral Word Association Test were not used to calculate the PCA because of the smaller number of individuals who completed these tests. In exploratory analyses, we also examined he association of each BP measure with individual test scores; the goal of these analyses was to better understand which component tests contributed the most to any observed associations.

\section{Statistical Analysis}

Descriptive characteristics of the study population were described by quartiles of DBP with frequency count and proportions for categorical and binary variables, means with SDs for continuous normally distributed variables, and medians with interquartile ranges for skewed variables.
We used random effects mixed models, accounting for the correlation between repeated measures, to explore the change in cognitive test scores over time. We postulated a linear model with a random intercept and slope for each cognitive test in which at time $j$ for the $i$ th patient, the cognitive test $\mathrm{Y}_{\mathrm{ij}}$ is given by, $\mathrm{Y}_{\mathrm{ij}}=$ $\left(\beta_{1}+\mathrm{b}_{1 \mathrm{i}}\right)+\left(\beta_{2}+\mathrm{b}_{2 \mathrm{i}}\right) \mathrm{t}_{\mathrm{j}}+\varepsilon_{\mathrm{ij}} ; i=1 ; \ldots ; \mathrm{n} ; j=1 ; \ldots ; \mathrm{n}_{\mathrm{i}}$, where $\beta_{1}$ and $\beta_{2}$ are the population mean intercept and rate of change. Because of the high rate of dropout due to mortality and transplant, and the fact that mortality and other causes of dropout may be informative, such that cognitive decline may have been greater in those who die before subsequent testing, we used shared random effects joint models for repeated measures and time to event data to estimate the rate of change in cognitive function decline adjusting for informative censoring due to mortality, transplant, or other reasons [21]. The joint models approach includes a linear mixed submodel for the repeated measurement and a survival submodel for time to dropout. These 2 models are linked using a shared random effect to account for noninformative dropout. The survival submodel included was a stratified cause-specific Cox competing risk model with 3 possible endpoints: (1) mortality, which also included dropout due to prolonged hospitalization or being too sick to complete testing; (2) transplant, transfer to peritoneal dialysis or transfer to a nonparticipating facility; and (3) no dropout. We separated out these causes of dropout to account for the fact that the association of the most proximate cognitive test with mortality and transplant could be in opposite directions.

To explore the association of each BP measure with the rate of change of the cognitive test, the linear mixed submodel included the main effect of the covariate of interest, time since baseline as a linear term, and their product. The time effect provides the slope of the cognitive test for the reference level of the covariate, and the interaction term provides the increase/decrease in this slope for the other level of the covariate. Models were initially unadjusted and subsequently adjusted for baseline age, sex, race, education level, cause of ESRD, dialysis vintage, and history of CVD. The competing risk survival submodel was adjusted for the same covariates included in the linear mixed submodel in addition to variables previously shown to be associated with mortality including vascular access type, serum albumin, BMI, and spKt/V [22]. To explore potential nonlinear relationships, we divided the cohort into quartiles for each BP measure and examined the relationship between each quartile with cognitive decline. Plots were created to demonstrate the rate of decline for each quartile of BP measures, adjusting for the same covariates described above. Figure 1 represent predicted trajectories for 4 individuals with levels equaling the mean BP measure within each quartile. Finally, to explore if the effect of BP parameters on the intercept and rate of change of the cognitive test is deviant from linear in favor of a U-shaped effect, we also included in our multivariable model the square of the BP term as an additional main effect and also as an additional interaction term with follow-up time.

Significance was assessed using a 2-sided alpha of 0.05 , and results were presented with 95\% CIs. All analyses were performed using SAS Enterprise Guide (version 7.12, Cary, NC, USA) and R language version 3.3.1 (R Foundation for Statistical Computing, Vienna Austria). Random effects models were fitted using the lme function in $\mathrm{R}$ package nlme. The joint models were estimated using the JM package in R (version JM 1.4-4) [23]. 


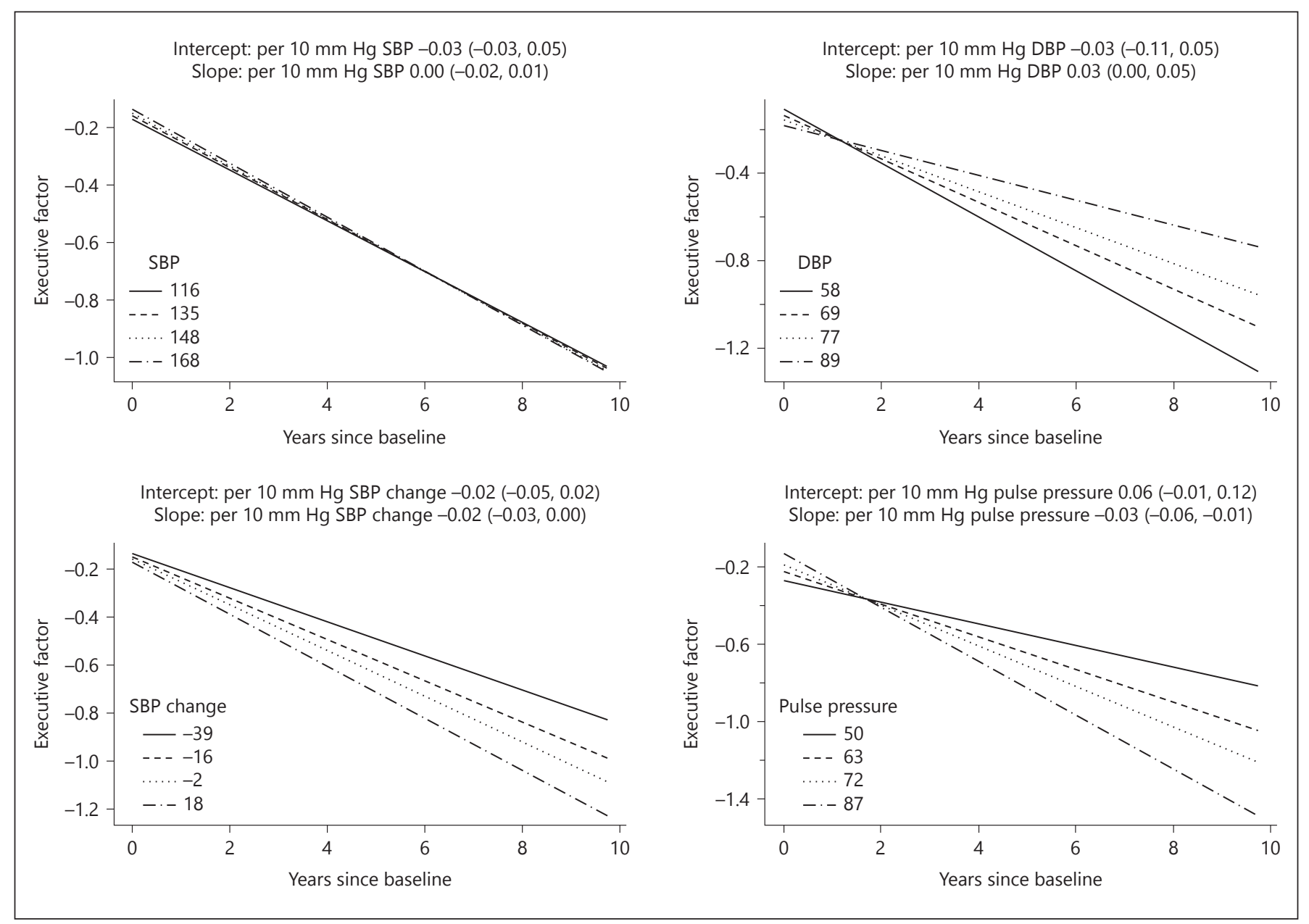

Fig. 1. Figure shows the predicted trajectories of executive function over time for 4 modeled subjects with levels equaling the mean blood pressure measure within each quartile. Adjusted for baseline age, sex, race, education level, cause of ESRD, dialysis vintage, and history of CVD. The competing risk survival submodel was ad-

\section{Results}

\section{Baseline Characteristics}

Among the 929 patients screened, 414 were ineligible for complete cognitive testing. Reasons included language barriers $(n=194,47 \%)$; behavioral and psychiatric issues $(n=14,3 \%)$; physical impediments such as blindness, hearing loss, and paralysis $(n=62,15 \%)$; recent acute hospitalization or impending death $(n=108$, $26 \%)$; mention of dementia in the medical record $(n=35,8 \%)$; and a score on the MMSE of $<10(n=1$, $<1 \%)$. Of the remaining 515,314 individuals consented to and underwent detailed cognitive testing [6]. Three hundred participants had sufficient data to calculate justed for the same covariates included in the linear mixed submodel in addition to variables previously shown to be associated with mortality including vascular access type, serum albumin, $\mathrm{BMI}$, and spKt/V. Slope values are presented as per $10 \mathrm{~mm} \mathrm{Hg}$ change in blood pressure measure.

memory and executive principal components. Table 1 describes the demographics and clinical characteristics of the whole study group and by quartiles of DBP. The mean (SD) age of study participants at enrollment was 62 (17) years; $54 \%$ were men, $23 \%$ were black, and $90 \%$ had at least a high school education (Table 1). During median follow-up of 2.1 years (25th-75th: 1.0-4.5), 191 (64\%) had at least one follow-up test with median number of follow-up tests of 2 (25th-75th: 1-4), while 148 (49\%) died during the study, 43 (14\%) received a kidney transplant and 39 (13\%) dropped out because of modality change or transfer to another dialysis unit. Across quartiles of DBP, those with lower DBP were more likely to be older and non-black and were more likely to 
Table 1. Baseline characteristics by quartile of DBP

\begin{tabular}{|c|c|c|c|c|c|c|}
\hline & $\begin{array}{l}\text { Total cohort } \\
(n=300)\end{array}$ & $\begin{array}{l}\text { Quartile } 1 \\
(n=74)\end{array}$ & $\begin{array}{l}\text { Quartile } 2 \\
(n=70)\end{array}$ & $\begin{array}{l}\text { Quartile } 3 \\
(n=80)\end{array}$ & $\begin{array}{l}\text { Quartile } 4 \\
(n=76)\end{array}$ & $\begin{array}{l}\text { Trend } \\
p \text { value }\end{array}$ \\
\hline Pre DBP monthly average, $\mathrm{mm} \mathrm{Hg}$ & $73.5 \pm 12.2$ & $58.6 \pm 4.5$ & $68.7 \pm 2.4$ & $76.6 \pm 2.7$ & $89.1 \pm 7.7$ & $<0.0001$ \\
\hline Age, years & $62.4 \pm 16.6$ & $71.4 \pm 12.0$ & $66.9 \pm 14.5$ & $60.2 \pm 17.1$ & $51.9 \pm 15.3$ & $<0.0001$ \\
\hline Female & $138(46.0)$ & $33(44.6)$ & $30(42.9)$ & $37(46.3)$ & $38(50.0)$ & 0.45 \\
\hline Black & $68(22.7)$ & $7(9.5)$ & $11(15.7)$ & $22(27.5)$ & $28(36.8)$ & $<0.0001$ \\
\hline Education & & & & & & 0.81 \\
\hline$<12$ th grade & $29(9.7)$ & $5(6.8)$ & $11(15.7)$ & $8(10.0)$ & $5(6.6)$ & \\
\hline High school graduate & $165(55.0)$ & $42(56.8)$ & $36(51.4)$ & $48(60.0)$ & $39(51.3)$ & \\
\hline$>2$ years college & $106(35.3)$ & $27(36.5)$ & $23(32.9)$ & $24(30.0)$ & $32(42.1)$ & \\
\hline Pre SBP monthly average, $\mathrm{mm} \mathrm{Hg}$ & $141.3 \pm 20.8$ & $121.9 \pm 16.7$ & $137.7 \pm 13.7$ & $145.5 \pm 16.2$ & $159.0 \pm 17.1$ & $<0.001$ \\
\hline Pulse pressure, $\mathrm{mm} \mathrm{Hg}$ & $67.8 \pm 14.7$ & $63.4 \pm 14.5$ & $69.0 \pm 13.9$ & $69.0 \pm 15.3$ & $69.9 \pm 14.3$ & 0.01 \\
\hline Post-pre SBP, mm Hg & $-10.0 \pm 23.5$ & $0.2 \pm 18.7$ & $-7.9 \pm 24.3$ & $-12.3 \pm 22.0$ & $-19.5 \pm 24.6$ & $<0.0001$ \\
\hline Stroke & $51(17.0)$ & $14(18.9)$ & $14(20.0)$ & $11(13.8)$ & $12(15.8)$ & 0.42 \\
\hline Peripheral vascular disease & $69(23.0)$ & $26(35.1)$ & $19(27.1)$ & $12(15.0)$ & $12(15.8)$ & 0.001 \\
\hline Diabetes & $143(47.7)$ & $38(51.4)$ & $42(60.0)$ & $39(48.8)$ & $24(31.6)$ & 0.007 \\
\hline Heart failure & $106(35.3)$ & $29(39.2)$ & $30(42.9)$ & $25(31.3)$ & $22(29.0)$ & 0.09 \\
\hline Coronary artery disease & $111(37.0)$ & $43(58.1)$ & $32(45.7)$ & $21(26.3)$ & $15(19.7)$ & $<0.0001$ \\
\hline Primary cause of ESRD & & & & & & 0.004 \\
\hline Diabetes & $102(34.0)$ & $33(44.6)$ & $29(41.4)$ & $24(30.0)$ & $16(21.1)$ & \\
\hline Hypertension & $57(19.0)$ & $13(17.6)$ & $15(21.4)$ & $12(15.0)$ & $17(22.4)$ & \\
\hline Other & $50(16.7)$ & $7(9.5)$ & $7(10.0)$ & $18(22.5)$ & $18(23.7)$ & \\
\hline Unknown & $39(13.0)$ & $9(12.2)$ & $11(15.7)$ & $9(11.3)$ & $10(13.2)$ & \\
\hline Glomerulonephritis & $52(17.3)$ & $12(16.2)$ & $8(11.4)$ & $17(21.3)$ & $15(19.7)$ & \\
\hline Dialysis vintage, months & $14.4(7.0-35.4)$ & $12.7(6.5-30.4)$ & $18.1(8.1-40.0)$ & $13.4(6.4-26.0)$ & $16.9(6.9-51.5)$ & 0.25 \\
\hline Vascular access & & & & & & 0.6 \\
\hline Fistula & $194(64.7)$ & $47(63.5)$ & $49(70.0)$ & $55(68.8)$ & $43(56.6)$ & \\
\hline Graft & $18(6.0)$ & $4(5.4)$ & $6(8.6)$ & $3(3.8)$ & $5(6.6)$ & \\
\hline Catheter & $88(29.3)$ & $23(31.1)$ & $15(21.4)$ & $22(27.5)$ & $28(36.8)$ & \\
\hline $\mathrm{BMI}, \mathrm{kg} / \mathrm{m}^{2}$ & $28.1 \pm 6.5$ & $27.8 \pm 6.1$ & $29.7 \pm 7.3$ & $28.2 \pm 6.2$ & $26.9 \pm 6.1$ & 0.22 \\
\hline $\mathrm{Kt} / \mathrm{V}$ & $1.51 \pm 0.24$ & $1.53 \pm 0.23$ & $1.52 \pm 0.23$ & $1.47 \pm 0.23$ & $1.53 \pm 0.27$ & 0.37 \\
\hline Albumin, g/dL & $3.8 \pm 0.4$ & $3.7 \pm 0.3$ & $3.8 \pm 0.3$ & $3.8 \pm 0.4$ & $3.9 \pm 0.4$ & 0.002 \\
\hline
\end{tabular}

Data are presented either as $n(\%)$, mean \pm SD, or median (25th, 75th percentiles).

BMI, body mass index; BP, blood pressure; SBP, systolic blood pressure; DBP, diastolic blood pressure.

have history of CVD (peripheral vascular disease and/or coronary disease) and diabetes (Table 1). Other demographics and clinical characteristics were similar across DBP quartiles.

Similar results were seen for linear mixed models and joint models. Therefore, as the joint models account for potential informative censoring from death or kidney transplantation, only joint model findings are described below.

\section{Diastolic Blood Pressure}

Lower DBP was a significant risk factor for steeper decline in the executive function summary score but not for the memory score (Table 2). Lower predialysis DBP was also a significant risk factor for steeper cognitive decline across multiple cognitive tests (Table 2), the majority of which assess executive function. Similar patterns were seen in quartile analysis (Fig. 1), with the lowest quartile of DBP measures associated with the steepest decline in executive function, while no patterns were observed for memory (online suppl. Fig. 1). For example, after 5 years a participant in the lowest DBP quartile (average diastolic $\mathrm{BP}$ of $58 \mathrm{~mm} / \mathrm{Hg}$ ) would on average have a $0.4 \mathrm{SD}$ lower executive function score compared to a participant in the highest diastolic BP quartile (average diastolic BP of $89 \mathrm{~mm} / \mathrm{Hg}$ ).

\section{Systolic Blood Pressure}

Higher predialysis SBP was not a risk factor for cognitive decline in unadjusted analyses (Table 3). This finding was consistent in multivariable analyses and in quartile analyses (Fig. 1; online suppl. Fig. 1). SBP was similarly not associated with steeper decline in any of the component cognitive tests (Table 2).

\section{Pulse Pressure}

Higher pulse pressure was associated with steeper decline in executive function in both unadjusted 
Table 2. Association between DBP (per $10 \mathrm{~mm} \mathrm{Hg}$ higher) and difference in slope of cognitive tests (per year change)

\begin{tabular}{|c|c|c|c|c|c|c|c|c|}
\hline Type of test & Cognitive test & Scoring & $n$ & Number & Unadjusted slope ${ }^{\wedge}$ & $p$ value & Adjusted slope (95\% CI) & $p$ value \\
\hline \multirow[t]{2}{*}{ Summary score } & Memory & $\mathrm{SD}^{*}$ & 300 & 645 & $0.01(-0.01$ to 0.04$)$ & 0.2 & $0.01(-0.01$ to 0.04$)$ & 0.2 \\
\hline & Executive function & $\mathrm{SD}^{*}$ & 300 & 645 & $0.03(0.00$ to 0.05$)$ & 0.03 & $0.03(0.00$ to 0.05$)$ & 0.03 \\
\hline Screening & MMSE & No. correct & 314 & 785 & $0.06(-0.06$ to 0.17$)$ & 0.3 & $0.05(-0.06$ to 0.16$)$ & 0.4 \\
\hline \multirow[t]{3}{*}{ Primarily memory } & Short delayed recall & No. correct & 312 & 779 & $0.01(-0.06$ to 0.08$)$ & 0.8 & $0.01(-0.06$ to 0.08$)$ & 0.8 \\
\hline & Delayed recall & No. correct & 312 & 774 & $0.10(0.04$ to 0.17$)$ & $<0.01$ & $0.10(0.04$ to 0.16$)$ & $<0.001$ \\
\hline & Word recognition & No. correct & 312 & 775 & $0.05(-0.01$ to 0.11$)$ & 0.1 & $0.05(-0.01$ to 0.11$)$ & 0.1 \\
\hline \multirow{9}{*}{$\begin{array}{l}\text { Primarily executive } \\
\text { function }\end{array}$} & Block design & No. completed & 311 & 752 & $0.32(0.08$ to 0.56$)$ & $<0.01$ & $0.30(0.07$ to 0.52$)$ & $<0.01$ \\
\hline & Digit symbol substitution & No. completed & 296 & 676 & $0.63(0.29$ to 0.96$)$ & $<0.001$ & $0.58(0.27$ to 0.88$)$ & $<0.001$ \\
\hline & Digits forward & No. correct & 187 & 452 & $-0.03(-0.09$ to 0.03$)$ & 0.3 & $-0.02(-0.08$ to 0.04$)$ & 0.4 \\
\hline & Digits backward & No. correct & 186 & 451 & $0.03(-0.03$ to 0.10$)$ & 0.3 & $0.04(-0.02$ to 0.11$)$ & 0.2 \\
\hline & Trail making part A & Time to completion, $s$ & 302 & 693 & $-1.53(-3.86$ to 0.79$)$ & 0.2 & $-1.43(-3.71$ to 0.85$)$ & 0.2 \\
\hline & Trail making part B & Time to completion, $s$ & 299 & 681 & $-2.06(-4.26$ to 0.14$)$ & 0.07 & $-1.94(-3.96$ to 0.09$)$ & 0.1 \\
\hline & Mental alternations & No. correct & 187 & 453 & 0.03 ( -0.17 to 0.22$)$ & 0.8 & 0.08 (-0.11 to 0.27$)$ & 0.4 \\
\hline & COWAT (animals) & No. correct & 187 & 453 & $0.11(-0.05$ to 0.28$)$ & 0.2 & $0.14(-0.02$ to 0.31$)$ & 0.1 \\
\hline & COWAT (supermarket) & No. correct & 187 & 454 & $0.22(0.03$ to 0.41$)$ & 0.02 & $0.25(0.07$ to 0.44$)$ & $<0.01$ \\
\hline
\end{tabular}
tests.

^Slope is the rate of change in the cognitive test per year. ests.

Summary scores represent a per SD change, with a mean of 0 and SD of 1 . Negative coefficients are associated with steeper decline in scores over time except on the trail making

Bold indicates statistically significant differences over time.

$n=$ number of unique patients and $N=$ number of follow-up visits. The digits, mental alternations, and COWAT tests were added halfway through study enrollment.

MMSE, mini-mental state examination; COWAT, controlled oral word association test; DBP, diastolic blood pressure.

Table 3. Association between SBP (per $10 \mathrm{~mm} \mathrm{Hg}$ higher) and difference in slope of cognitive tests (per year change)

\begin{tabular}{|c|c|c|c|c|c|c|c|c|}
\hline Type of test & Cognitive test & Scoring & $n$ & Number & Unadjusted slope ${ }^{\wedge}$ & $p$ value & Adjusted slope $(95 \% \mathrm{CI})$ & $p$ value \\
\hline \multirow[t]{2}{*}{ Summary score } & Memory & $\mathrm{SD}^{*}$ & 300 & 645 & $0.00(-0.01$ to 0.02$)$ & 0.5 & $0.00(-0.01$ to 0.02$)$ & 0.8 \\
\hline & Executive function & $\mathrm{SD}^{*}$ & 300 & 645 & $0.00(-0.02$ to 0.02$)$ & 0.9 & $0.00(-0.02$ to 0.01$)$ & 0.8 \\
\hline Screening & MMSE & No. correct & 314 & 785 & $-0.03(-0.10$ to 0.04$)$ & 0.4 & $-0.03(-0.11$ to 0.04$)$ & 0.3 \\
\hline \multirow[t]{3}{*}{ Primarily memory } & Short delayed recall & No. correct & 312 & 779 & $-0.01(-0.06$ to 0.04$)$ & 0.8 & $-0.01(-0.06$ to 0.04$)$ & 0.6 \\
\hline & Delayed recall & No. correct & 312 & 774 & $0.03(-0.01$ to 0.07$)$ & 0.2 & $0.02(-0.02$ to 0.06$)$ & 0.2 \\
\hline & Word recognition & No. correct & 312 & 775 & $0.01(-0.04$ to 0.06$)$ & 0.7 & $0.01(-0.04$ to 0.06$)$ & 0.8 \\
\hline \multirow{9}{*}{$\begin{array}{l}\text { Primarily executive } \\
\text { function }\end{array}$} & Block design & No. completed & 311 & 752 & $0.10(-0.06$ to 0.26$)$ & 0.2 & $0.08(-0.07$ to 0.23$)$ & 0.3 \\
\hline & Digit symbol substitution & No. completed & 296 & 676 & $0.11(-0.14$ to 0.35$)$ & 0.4 & $0.07(-0.16$ to 0.29$)$ & 0.6 \\
\hline & Digits forward & No. correct & 187 & 452 & $0.01(-0.04$ to 0.05$)$ & 0.8 & $0.01(-0.04$ to 0.05$)$ & 0.8 \\
\hline & Digits backward & No. correct & 186 & 451 & $0.02(-0.03$ to 0.06$)$ & 0.5 & $0.02(-0.03$ to 0.06$)$ & 0.4 \\
\hline & Trail making part A & Time to completion (s) & 302 & 693 & $0.90(-0.59$ to 2.40$)$ & 0.2 & $0.97(-0.50$ to 2.44$)$ & 0.2 \\
\hline & Trail making part B & Time to completion (s) & 299 & 681 & $0.30(-1.21$ to 1.80$)$ & 0.7 & $0.34(-0.99$ to 1.68$)$ & 0.6 \\
\hline & Mental alternations & No. correct & 187 & 453 & $-0.01(-0.15$ to 0.13$)$ & 0.9 & $0.02(-0.12$ to 0.15$)$ & 0.8 \\
\hline & COWAT (animals) & No. correct & 187 & 453 & $0.13(0.02$ to 0.24$)$ & 0.03 & $0.13(0.02$ to 0.24$)$ & 0.02 \\
\hline & COWAT (supermarket) & No. correct & 187 & 454 & $0.07(-0.09$ to 0.22$)$ & 0.4 & $0.07(-0.07$ to 0.22$)$ & 0.3 \\
\hline
\end{tabular}

^Slope is the rate of change in the cognitive test per year.

* Summary scores represent a per SD change. Negative coefficients are associated with steeper decline in scores over time except on the trail making tests.

Bold indicates statistically significant differences over time.

$n=$ number of unique patients and $N=$ number of follow-up visits.

The digits, mental alternations, and COWAT tests were added halfway through study enrollment.

MMSE, mini-mental state examination; COWAT, controlled oral word association test; SBP, systolic blood pressure.

and adjusted analyses; this relationship was not observed for memory (Table 4). High pulse pressure was also associated with steeper decline in several individual cognitive tests, including both trailmaking tests. A similar pattern of higher pulse pressure associated with steeper decline in executive function but not memory was seen in quartile analyses (Fig. 1; online suppl. Fig. 1).

$\mathrm{BP}$ and Cognitive Decline in Prevalent HD Patients

\section{Intradialytic Change in $B P$}

An increase in SBP after the HD treatment was associated with steeper decline in executive function but not memory in unadjusted analyses (Table 5). This association was attenuated and became nonsignificant after adjustment. Similarly, an increase in SBP after HD was associated with steeper decline in 3 individual tests; however, after adjustment, only the association with the Word 
Table 4. Association between pulse pressure (per $10 \mathrm{~mm} \mathrm{Hg}$ higher) and difference in slope of cognitive tests (per year change)

\begin{tabular}{|c|c|c|c|c|c|c|c|c|}
\hline Type of test & Cognitive test & Scoring & $n$ & Number & Unadjusted slope ${ }^{\wedge}$ & $p$ value & $\begin{array}{l}\text { Adjusted slope } \\
(95 \% \text { CI })\end{array}$ & $p$ value \\
\hline \multirow[t]{2}{*}{ Summary score } & Memory & $\mathrm{SD}^{*}$ & 300 & 645 & $0.00(-0.03$ to 0.02$)$ & 0.8 & $0.00(-0.03$ to 0.02$)$ & 0.8 \\
\hline & Executive function & $\mathrm{SD}^{*}$ & 300 & 645 & $-0.04(-0.06$ to -0.01$)$ & $<0.01$ & $-0.03(-0.06$ to -0.01$)$ & $<0.01$ \\
\hline Screening & MMSE & No. correct & 314 & 785 & $-0.13(-0.24$ to -0.02$)$ & 0.02 & $-0.13(-0.24$ to -0.02$)$ & 0.02 \\
\hline \multirow{3}{*}{ Primarily memory } & Short delayed recall & No. correct & 312 & 779 & $-0.03(-0.11$ to 0.04$)$ & 0.4 & $-0.03(-0.11$ to 0.04$)$ & 0.4 \\
\hline & Delayed recall & No. correct & 312 & 774 & $-0.05(-0.13$ to 0.02$)$ & 0.2 & $-0.05(-0.12$ to 0.02$)$ & 0.2 \\
\hline & Word recognition & No. correct & 312 & 775 & $-0.03(-0.11$ to 0.04$)$ & 0.4 & $-0.03(-0.11$ to 0.04$)$ & 0.4 \\
\hline \multirow{9}{*}{$\begin{array}{l}\text { Primarily executive } \\
\text { function }\end{array}$} & Block design & No. completed & 311 & 752 & $-0.13(-0.38$ to 0.12$)$ & 0.3 & $-0.12(-0.35$ to 0.11$)$ & 0.3 \\
\hline & Digit symbol substitution & No. completed & 296 & 676 & $-0.50(-0.88$ to -0.12$)$ & 0.01 & $-0.51(-0.87$ to -0.16$)$ & $<0.01$ \\
\hline & Digits forward & No. correct & 187 & 452 & $0.04(-0.03$ to 0.11$)$ & 0.3 & $0.04(-0.03$ to 0.10$)$ & 0.3 \\
\hline & Digits backward & No. correct & 186 & 451 & $-0.01(-0.08$ to 0.07$)$ & 0.9 & $0.00(-0.07$ to 0.07$)$ & $>0.99$ \\
\hline & Trail making part A & Time to completion, $\mathrm{s}$ & 302 & 693 & $3.42(1.25$ to 5.58$)$ & $<0.01$ & $3.49(1.33$ to 5.65$)$ & $<0.01$ \\
\hline & Trail making part $\mathrm{B}$ & Time to completion, $s$ & 299 & 681 & $3.24(0.98$ to 5.50$)$ & $<0.01$ & $2.83(0.74$ to 4.93$)$ & $<0.01$ \\
\hline & Mental alternations & No. correct & 187 & 453 & $-0.09(-0.31$ to 0.12$)$ & 0.4 & $-0.05(-0.26$ to 0.15$)$ & 0.6 \\
\hline & COWAT (animals) & No. correct & 187 & 453 & $0.15(-0.03$ to 0.32$)$ & 0.1 & $0.14(-0.03$ to 0.31$)$ & 0.1 \\
\hline & COWAT (supermarket) & No. correct & 187 & 454 & $-0.14(-0.38$ to 0.10$)$ & 0.2 & $-0.11(-0.31$ to 0.09$)$ & 0.3 \\
\hline
\end{tabular}

\footnotetext{
^Slope is the rate of change in the cognitive test per year.

* Summary scores represent a per SD change, with a mean of 0 and SD of 1 .

Negative coefficients are associated with steeper decline in scores over time except on the trail making tests.

Bold indicates statistically significant differences over time.

$n=$ number of unique patients and $N=$ number of follow-up visits. The digits, mental alternations, and COWAT tests were added halfway through study enrollment.

MMSE, mini-mental state examination; COWAT, controlled oral word association test.
}

Recognition test remained significant. In quartile analyses, those participants with a rise in SBP after HD were more likely to have executive function decline compared to those who experienced a fall in SBP.

\section{"U" Shaped Relationships}

There was no evidence of a " $U$ " shaped relationship between any of the BP measures and decline in the composite memory or executive function score (all $p$ values $>0.05)$.

\section{Discussion}

Lower predialysis DBP and higher pulse pressure were both associated with steeper decline in cognitive function in maintenance HD patients. SBP, in contrast, showed no association with cognitive decline, indicating that low DBP is likely the component of pulse pressure that carries prognostic importance. The associations with decline were primarily seen with the executive function summary score and the component tests that comprise the executive function domain rather than with tests that assess memory. The observed difference in slopes leads to substantial changes in cognitive function over time. For those in the lowest diastolic quartile (or highest pulse pressure quartile), this translates to nearly a half SD difference in test scores after 5 years. The difference in how fast participants with differing BPs decline appears significant viewed in comparison to the general population. For example, one such study in the general population reported a decline in MMSE score of 0.02 per year for participants aged 65 years and a decline in MMSE score of 0.57 per year for participants aged 85 years [24]. In comparison, individuals in this study with a pulse pressure in the highest quartile (mean difference in systolic and diastolic $\mathrm{BP}=87 \mathrm{~mm} \mathrm{Hg}$ ) experienced a decline in the MMSE of 0.48 per year greater than those in the lowest quartile (mean difference in systolic and diastolic $\mathrm{BP}=$ $50 \mathrm{~mm} \mathrm{Hg}$ ).

In the general population, systolic and diastolic hypertension are associated with cognitive impairment [2], cognitive function decline [3], and incident dementia [4]. At least one study has also reported a nonlinear, "U"shaped relationship between BP and cognitive function [25]. An analysis of the Baltimore Longitudinal Study of Aging demonstrated that both high and low DBPs were associated with poorer executive function compared to normotensive individuals. It is less clear how these findings translate to those individuals with $\mathrm{CKD}$, particularly those requiring maintenance $\mathrm{HD}$, given the associations between low BP and higher mortality in this population [26-28].

There is a substantial literature exploring the associations between BP and mortality in patients receiving maintenance HD. An analysis of approximately 11,000 maintenance HD patients from the United States Renal 
Table 5. Association between intra-dialytic SBP change (per $10 \mathrm{~mm} \mathrm{Hg}$ higher) and difference in slope of cognitive tests (per year change)

\begin{tabular}{|c|c|c|c|c|c|c|c|c|}
\hline Type of test & Cognitive test & Scoring & $n$ & Number & Unadjusted slope & $p$ value & Adjusted slope $(95 \%$ CI) & $p$ value \\
\hline \multirow[t]{2}{*}{ Summary score } & Memory & $\mathrm{SD}^{*}$ & 300 & 645 & $0.00(-0.02$ to 0.01$)$ & 0.7 & $0.00(-0.02$ to 0.01$)$ & 0.7 \\
\hline & Executive function & $\mathrm{SD}^{*}$ & 300 & 645 & $-0.02(-0.03$ to 0.00$)$ & 0.04 & $-0.02(-0.03$ to 0.00$)$ & 0.1 \\
\hline Screening & MMSE & No. correct & 314 & 785 & $-0.01(-0.08$ to 0.06$)$ & 0.8 & $-0.01(-0.07$ to 0.06$)$ & 0.9 \\
\hline \multirow[t]{3}{*}{ Primarily memory } & Short delayed recall & No. correct & 312 & 779 & $-0.02(-0.07$ to 0.03$)$ & 0.4 & $-0.02(-0.06$ to 0.02$)$ & 0.3 \\
\hline & Delayed recall & No. correct & 312 & 774 & $-0.03(-0.08$ to 0.02$)$ & 0.2 & $-0.03(-0.07$ to 0.01$)$ & 0.2 \\
\hline & Word recognition & No. correct & 312 & 775 & $-0.05(-0.10$ to -0.01$)$ & 0.03 & $-0.05(-0.09$ to -0.01$)$ & $<0.01$ \\
\hline \multirow{9}{*}{$\begin{array}{l}\text { Primarily executive } \\
\text { function }\end{array}$} & Block design & No. completed & 311 & 752 & $-0.18(-0.34$ to -0.02$)$ & 0.03 & $-0.15(-0.30$ to 0.00$)$ & 0.1 \\
\hline & Digit symbol substitution & No. completed & 296 & 676 & $-0.11(-0.35$ to 0.12$)$ & 0.4 & $-0.07(-0.29$ to 0.15$)$ & 0.5 \\
\hline & Digits forward & No. correct & 187 & 452 & $0.03(-0.02$ to 0.07$)$ & 0.2 & $0.03(-0.01$ to 0.07$)$ & 0.2 \\
\hline & Digits backward & No. correct & 186 & 451 & $0.00(-0.04$ to 0.05$)$ & $>0.99$ & $0.00(-0.04$ to 0.05$)$ & 0.9 \\
\hline & Trail making part A & Time to completion, s & 302 & 693 & $0.49(-0.86$ to 1.83$)$ & 0.5 & $0.44(-0.89$ to 1.77$)$ & 0.5 \\
\hline & Trail making part B & Time to completion, s & 299 & 681 & $0.92(-0.54$ to 2.38$)$ & 0.2 & $0.66(-0.69$ to 2.01$)$ & 0.3 \\
\hline & Mental alternations & No. correct & 187 & 453 & $0.04(-0.10$ to 0.18$)$ & 0.6 & 0.05 ( -0.09 to 0.18$)$ & 0.5 \\
\hline & COWAT (animals) & No. correct & 187 & 453 & $-0.12(-0.23$ to -0.01$)$ & 0.04 & $-0.10(-0.21$ to 0.01$)$ & 0.1 \\
\hline & COWAT (supermarket) & No. correct & 187 & 454 & $-0.07(-0.20$ to 0.06$)$ & 0.3 & $-0.05(-0.18$ to 0.08$)$ & 0.4 \\
\hline $\begin{array}{l}\text { ^Slope is the rat } \\
{ }^{*} \text { Summary scor } \\
\text { Negative coeffic } \\
\text { Bold indicates } \\
n=\text { number of } \\
\text { MMSE, mini-m }\end{array}$ & $\begin{array}{l}\text { change in the cognitive tes } \\
\text { present a per SD change, } \mathrm{x} \\
\text { are associated with steepe } \\
\text { ically significant difference } \\
\text { ee patients and } N=\text { numbe } \\
\text { state examination; COW }\end{array}$ & $\begin{array}{l}\text { ear. } \\
\text { mean of } 0 \text { and SD of } 1 . \\
\text { ine in scores over time } \\
\text { time. } \\
\text { llow-up visits. The digit } \\
\text { ntrolled oral word asso }\end{array}$ & 01 & trail mak & $\begin{array}{l}\text { tests. } \\
\text { COWAT tests were ac } \\
\text { lood pressure. }\end{array}$ & d halfu & hrough study enrollment. & \\
\hline
\end{tabular}

Data System Waves 3 and 4 study demonstrated that low predialysis systolic, low predialysis diastolic, and high post dialysis SBP were all independently associated with all-cause mortality [28]. The authors concluded that widened pulse pressure was the strongest risk factor all-cause mortality. An observational study of over 5,000 HD patients by Zager et al. [29] noted a " $U$ " shaped association between post dialysis SBP and cardiovascular specific mortality, while Robinson et al. [30] found that in a cohort of over 4,000 maintenance HD patients that low predialysis SBP carried the greatest risk of mortality compared to other BP measures. Finally, a study by Kovesdy et al. [31] of more than 650,000 US veterans with CKD found that those participants with low DBP $(>70 \mathrm{~mm} \mathrm{Hg}$ ) were at higher risk for all-cause mortality, even if the SBP was considered "ideal."

Relatively few studies have investigated the link between BP and cognitive function in patients with kidney disease. The Chronic Renal Insufficiency Cohort examined cross-sectional vascular risk factors for cognitive impairment in 3,591 participants with a mean eGFR of 43 $\mathrm{mL} / \mathrm{min} / 1.73 \mathrm{~m}^{2}$, finding that hypertension was a significant risk factor for cognitive impairment in unadjusted analyses but not after adjustment for kidney function [9]. A recent study by MacEwen et al. [32] in participants on maintenance HD investigated the interplay between BP, cerebral ischemia (measured using cranial near-infrared spectroscopy), and cognitive function. Among the 58 participants, hypotension was associated with cerebral ischemia, but not cognitive impairment; however, the small sample size was a limiting factor in drawing conclusions. In the same cohort of maintenance HD patients reported in this manuscript, we previously demonstrated an association between lower DBP and worse cognitive performance; however, we found that this relationship was attenuated and became nonsignificant when adjusting for demographics and comorbidity [12]. There are even less data on the impact of different BP goals on cognitive function in CKD patients, and longitudinal data on cognitive function from the recently completed SBP Intervention Trial are eagerly awaited [33].

There are several potential mechanisms that could explain the association between lower BP and cognitive decline in individuals with CKD. First, low BP may be a marker of vascular disease. An analysis that combined data from the Cardiovascular Health Study and the Atherosclerosis Risk in Communities study demonstrated that low SBP was a significant risk factor for stroke, but only in those participants with reduced kidney function [34]. Importantly, cerebrovascular disease most commonly produces a deficit within executive cognitive function, the type of impairment we found to be associated with lower BP in this study. Second, those with low diastolic and high pulse pressure at the start of $\mathrm{HD}$, a marker of loss of vascular elasticity [35], may be the most susceptible to intradialytic hypotension and impaired autoregulation and thus decreased cerebral perfusion [36]. A recent study by Polinder-Bos et al. [37] examined the effect of HD on cerebral blood flow as measured by 
positron emission tomography-computed tomography imaging in 12 elderly participants. Cerebral blood flow was found to decline by $10 \%$ during a HD treatment, suggesting that $\mathrm{HD}$ treatments may induce repetitive cerebral ischemic injury. Taken together, these lines of evidence may indicate that for patients receiving maintenance $\mathrm{HD}$, standard BP targets may not apply. Largescale randomized clinical trials, in which participants requiring $\mathrm{HD}$ are assigned to different $\mathrm{BP}$ targets, may be the only definitive method to determine optimal BP in this population.

The strengths of this study include use of an expansive neurocognitive battery, detailed ascertainment of potential confounding variables, and use of statistical modeling to account for the competing risk of death and kidney transplantation. There are also several limitations. The high mortality rate of our dialysis cohort limited the number of repeat cognitive measures, potentially reducing our ability to detect differences in cognitive decline; however, this mortality rate is consistent with the broader HD population and we accounted for the semi-competing risk of mortality (and the opposing risk of drop-out by transplantation) using joint models. We also included prevalent not incident HD patients, which may introduce selection bias to the cohort. Additionally, we excluded participants with either a reported history of dementia or a very low MMSE score $(<10)$, which may underestimate the effect size associated with difference in BP. Finally, our measure of BP was obtained in the dialysis unit as part of standard clinical care, not via a standardized protocol, such as is done in many clinical trials. As a result, variation in cuff size selection, participant positioning, and timing may contribute to inaccurate $\mathrm{BP}$ readings. Although we believe our use of the monthly mean $\mathrm{BP}$ reduces the impact of day to day BP variability, there is some evidence that home monitoring of $\mathrm{BP}$ in dialysis patients may provide a more accurate measure than in-center measures [38].
Low DBP and widened pulse pressure were both shown to be risk factors for cognitive decline in a cohort of maintenance HD patients. Declines were most prominent in tests of executive function, suggesting that the association of DBP and pulse pressure may be mediated through cerebrovascular disease. Additional research should focus on determining optimal DBP targets in HD patients. Future studies are needed to determine if these findings extend to patients with CKD who do not yet require HD.

\section{Disclosure Statement}

D.E.W. receives salary support from Dialysis Clinic, Inc.

\section{Funding Source}

The study was funded by the NIDDK through grants R21 DK068310 (M.J.S.), R01 DK078204 (M.J.S.), and K23DK105327 (D.A.D.) as well as through a grant from the Paul Teschan Research Fund of Dialysis Clinic, Inc. The funders of this study had no role in the study design, collection, analysis and interpretation, writing, or decision to submit the manuscript for publication.

\section{Author Contributions}

D.A.D., D.E.W., H.T., T.S., and M.J.S.: research idea and study design. T.S., S.D., and J.R.: data acquisition. D.A.D., D.E.W., A.G., H.T., T.S., and M.J.S.: data analysis/interpretation. H.T.: statistical analysis. D.E.W., T.S., and M.J.S.: supervision or mentorship. Each author contributed important intellectual content during manuscript drafting or revision and accepts accountability for the overall work by ensuring that questions pertaining to the accuracy or integrity of any portion of the work are appropriately investigated and resolved. D.A.D.: takes responsibility that this study has been reported honestly, accurately, and transparently; that no important aspects of the study have been omitted, and that any discrepancies from the study as planned (and, if relevant, registered) have been explained.

\section{References}

1 Elias MF, Goodell AL, Dore GA. Hypertension and cognitive functioning: a perspective in historical context. Hypertension. 2012 Aug;60(2):260-8.

2 Elias MF, Elias PK, Sullivan LM, Wolf PA, D'Agostino RB. Lower cognitive function in the presence of obesity and hypertension: the Framingham heart study. Int $\mathrm{J}$ Obes Relat Metab Disord. 2003 Feb;27(2):260-8.
3 van Swieten JC, Geyskes GG, Derix MM, Peeck BM, Ramos LM, van Latum JC, et al. Hypertension in the elderly is associated with white matter lesions and cognitive decline. Ann Neurol. 1991 Dec;30(6):82530.

4 Skoog I, Lernfelt B, Landahl S, Palmertz B, Andreasson LA, Nilsson L, et al. 15-year longitudinal study of blood pressure and de- mentia. Lancet. 1996 Apr;347(9009):11415.

5 Kurella Tamura M, Xie D, Yaffe K, Cohen DL, Teal V, Kasner SE, et al. Vascular risk factors and cognitive impairment in chronic kidney disease: the Chronic Renal Insufficiency Cohort (CRIC) study. Clin J Am Soc Nephrol. 2011 Feb;6(2):24856. 
6 Sarnak MJ, Tighiouart H, Scott TM, Lou KV, Sorensen EP, Giang LM, et al. Frequency of and risk factors for poor cognitive performance in hemodialysis patients. Neurology. 2013 Jan;80(5):471-80.

7 Whelton PK, Carey RM, Aronow WS, Casey DE Jr, Collins KJ, Dennison Himmelfarb C, et al. 2017 ACC/AHA/AAPA/ABC/ACPM/ AGS/APhA/ASH/ASPC/NMA/PCNA guideline for the prevention, detection, evaluation, and management of high blood pressure in adults: a report of the American College of Cardiology/American Heart Association Task Force on Clinical Practice Guidelines. J Am Coll Cardiol. 2018 May;71(19):e127-248.

8 Reisin E, Harris RC, Rahman M. Commentary on the 2014 BP guidelines from the panel appointed to the Eighth Joint National Committee (JNC 8). J Am Soc Nephrol. 2014 Nov; 25(11):2419-24.

9 Cheung AK, Rahman M, Reboussin DM, Craven TE, Greene T, Kimmel PL, et al.; SPRINT Research Group. Effects of intensive BP control in CKD. J Am Soc Nephrol. 2017 Sep; 28(9):2812-23.

10 Rocco MV, Sink KM, Lovato LC, Wolfgram DF, Wiegmann TB, Wall BM, et al.; SPRINT Research Group. Effects of Intensive Blood Pressure Treatment on Acute Kidney Injury Events in the Systolic Blood Pressure Intervention Trial (SPRINT). Am J Kidney Dis. 2018 Mar;71(3):352-61.

11 Levin NW, Kotanko P, Eckardt KU, Kasiske BL, Chazot C, Cheung AK, et al. Blood pressure in chronic kidney disease stage 5D-report from a Kidney Disease: improving Global Outcomes controversies conference. Kidney Int. $2010 \mathrm{Feb} ; 77(4): 273-84$.

12 Giang LM, Tighiouart H, Lou KV, Agganis B, Drew DA, Shaffi K, et al. Measures of blood pressure and cognition in dialysis patients. Hemodial Int. 2013 Jan;17(1):24-31.

13 Weiner DE, Scott TM, Giang LM, Agganis BT, Sorensen EP, Tighiouart H, et al. Cardiovascular disease and cognitive function in maintenance hemodialysis patients. Am J Kidney Dis. 2011 Nov;58(5):773-81.

14 Drew DA, Tighiouart H, Scott TM, Lou KV, Shaffi K, Weiner DE, et al. Cognitive performance before and during hemodialysis: a randomized cross-over trial. Nephron Clin Pract. 2013;124(3-4):151-8.

15 Folstein MF, Folstein SE, McHugh PR. "Minimental state". A practical method for grading the cognitive state of patients for the clinician. J Psychiatr Res. 1975 Nov;12(3):189-98.
16 Tulsky D, Zhu J, Lebetter M. Wechsler Adult Intelligence Scale-Third Edition (WAIS-III), Wechsler Memory Scale-Third Scale (WMSIII): Technical Manual. San Antonio: Harcourt Brace \& Company; 1997.

17 Heaton R, Grant I, Mathews C. Comprehensive Norms for an Expanded Halstead-Reitan Battery. Odessa: Psychological Assessment Resources Inc.; 1991.

18 Salib E, McCarthy J. Mental Alternation Test (MAT): a rapid and valid screening tool for dementia in primary care. Int J Geriatr Psychiatry. 2002 Dec;17(12):1157-61.

19 Benton A, Hamsher K. Multilingual Aphasia Examination. Iowa City: University of Iowa; 1978.

20 Heyer NJ, Bittner AC Jr, Echeverria D. Analyzing multivariate neurobehavioral outcomes in occupational studies: a comparison of approaches. Neurotoxicol Teratol. 1996 Jul-Aug;18(4):401-6.

21 Rizopoulos DJ. An R package for the joint modelling of longitudinal and time-to-event data. J Stat Softw. 2010;35(9):1-33.

22 Drew DA, Weiner DE, Tighiouart $\mathrm{H}$, Scott $\mathrm{T}$, Lou K, Kantor A, et al. Cognitive Function and All-Cause Mortality in Maintenance Hemodialysis Patients. Am J Kidney Dis. 2015 Feb;65(2):303-11.

23 Rizopoulos D. Joint models for longitudinal and time-to-event data: With applications in R. CRC Press; 2012.

24 Jacqmin-Gadda H, Fabrigoule C, Commenges D, Dartigues JF. A 5-year longitudinal study of the Mini-Mental State Examination in normal aging. Am J Epidemiol. 1997 Mar; 145(6):498-506.

25 Waldstein SR, Giggey PP, Thayer JF, Zonderman AB. Nonlinear relations of blood pressure to cognitive function: the Baltimore Longitudinal Study of Aging. Hypertension. 2005 Mar;45(3):374-9.

26 Sumida K, Molnar MZ, Potukuchi PK, Thomas F, Lu JL, Ravel VA, et al. Blood Pressure Before Initiation of Maintenance Dialysis and Subsequent Mortality. Am J Kidney Dis. 2017 Aug;70(2):207-17.

27 Kalantar-Zadeh K, Kilpatrick RD, McAllister CJ, Greenland S, Kopple JD. Reverse epidemiology of hypertension and cardiovascular death in the hemodialysis population: the 58th annual fall conference and scientific sessions. Hypertension. 2005 Apr;45(4):8117.

28 Foley RN, Herzog CA, Collins AJ; United States Renal Data System. Blood pressure and long-term mortality in United States hemodialysis patients: USRDS Waves 3 and 4 Study. Kidney Int. 2002 Nov;62(5):1784-90.

29 Zager PG, Nikolic J, Brown RH, Campbell MA, Hunt WC, Peterson D, et al. "U” curve association of blood pressure and mortality in hemodialysis patients. Medical Directors of Dialysis Clinic, Inc. Kidney Int. 1998 Aug; 54(2):561-9.

30 Robinson BM, Tong L, Zhang J, Wolfe RA, Goodkin DA, Greenwood RN, et al. Blood pressure levels and mortality risk among hemodialysis patients in the Dialysis Outcomes and Practice Patterns Study. Kidney Int. 2012 Sep;82(5):570-80.

31 Kovesdy CP, Bleyer AJ, Molnar MZ, Ma JZ, Sim JJ, Cushman WC, et al. Blood pressure and mortality in U.S. veterans with chronic kidney disease: a cohort study. Ann Intern Med. 2013 Aug;159(4):233-42.

32 MacEwen C, Sutherland S, Daly J, Pugh C, Tarassenko L. Relationship between hypotension and cerebral ischemia during hemodialysis. J Am Soc Nephrol. 2017 Aug;28(8):251120.

33 Weiner DE, Gaussoin SA, Nord J, Auchus AP, Chelune GJ, Chonchol M, et al.; SPRINT Study Research Group. Cognitive Function and Kidney Disease: Baseline Data From the Systolic Blood Pressure Intervention Trial (SPRINT). Am J Kidney Dis. 2017 Sep;70(3): $357-67$.

34 Weiner DE, Tighiouart H, Levey AS, Elsayed E, Griffith JL, Salem DN, et al. Lowest systolic blood pressure is associated with stroke in stages 3 to 4 chronic kidney disease. J Am Soc Nephrol. 2007 Mar;18(3):960-6.

35 Briet M, Boutouyrie P, Laurent S, London GM. Arterial stiffness and pulse pressure in CKD and ESRD. Kidney Int. 2012 Aug;82(4): 388-400.

36 O’Rourke MF, Safar ME. Relationship between aortic stiffening and microvascular disease in brain and kidney: cause and logic of therapy. Hypertension. 2005 Jul;46(1): 200-4.

37 Polinder-Bos HA, García DV, Kuipers J, Elting JW, Aries MJ, Krijnen WP, et al. Hemodialysis Induces an Acute Decline in Cerebral Blood Flow in Elderly Patients. J Am Soc Nephrol. 2018 Apr;29(4):1317-25.

38 Agarwal R, Andersen MJ, Bishu K, Saha C. Home blood pressure monitoring improves the diagnosis of hypertension in hemodialysis patients. Kidney Int. 2006 Mar;69(5):9006. 